\title{
Actividades emprendedoras y espacios alternativos de aprendizaje. Desarrollo de competencias durante un hackathon day
}

\section{Entrepreneurial activities and alternative learning spaces. Skills development for a hackathon day}

\author{
Carmen Ruiz Viñals. Universidad Abat Oliba CEU (cruizv@uao.es) \\ Eva Perea Muñoz. Universidad Abat Oliba CEU (eperea@uao.es) \\ Vanesa Berlanga Silvente. Universidad Abat Oliba CEU (vberlanga@uao.es)
}

Resumen:

El proyecto de los espacios sociales de aprendizaje en la Universidad Española se inició como parte del cambio de modelo educativo propuesto por el proceso de Bolonia. Dichos espacios los conforman todos los lugares que no son los aularios y que ayudan a completar el aprendizaje formal. En la presente investigación se reflexiona sobre estos espacios y las actividades que se desarrollan en ellos, donde los estudiantes adquieren las competencias y habilidades más demandadas por el mercado laboral, especialmente las llamadas competencias emprendedoras. Anualmente se organiza un maratón de emprendimiento llamado Barcelona Thinking Challenge en la UAO CEU. Dicha actividad es un espacio de aprendizaje donde los estudiantes adquieren competencias transversales y específicas, trabajando en equipos formados por estudiantes de diferentes cursos, universidades y profesionales. Se analizan los resultados de un estudio de encuesta que valora cuál ha sido la visión general de este maratón de emprendimiento social y qué competencias emprendedoras ha permitido desarrollar en los participantes. Los resultados permiten destacar que los partícipes valoran positivamente esta actividad emprendedora. Se muestra un alto desarrollo en las competencias relacionadas con trabajar en equipo, liderazgo y aplicación de conocimientos adquiridos en la universidad a la resolución de problemas.

Palabras clave:

Actividades emprendedoras; empleabilidad; modalidades de aprendizaje; innovación social; espacios alternativos de aprendizaje Abstract:

The promotion of social learning spaces is part of the transformation in the educational model proposed by the Bologna process. Social learning spaces are all of the areas that are not classrooms, and which help students complete their formal education. This research reflects on social learning spaces and the activities that are developed therein where students acquire the skills demanded by the labor market, especially those known as entrepreneurial competences. The Abat Oliba CEU University organizes an annual marathon venture called Barcelona Thinking Challenge. This activity takes place in a social learning space where students acquire transversal and specific skills, as they have the opportunity to work in teams composed of students from different courses and universities, as well as company professionals. This research analyzes the results of a quantitative study that describes the students' assessment of the activity. It also tries to determine what entrepreneurial skills the activity has allowed the participants to develop. The findings show that participants place a high value on the entrepreneurial marathon. The results also demonstrate a strong development of skills related to working in teams, leadership, and applying knowledge acquired at university to problem-solving tasks. Therefore, oral communication of ideas, problems and solutions is one of the skills that participants valued most during the marathon.

Key words:

Entrepreneurial activities; employability; learning modalities; social innovation; alternative learning spaces 


\section{Introducción}

La estrategia española para la modernización de las universidades se ha centrado en conseguir la mejora en tres objetivos generales y transversales: la excelencia, la internacionalización y la promoción de la dimensión social y la empleabilidad. Ello se ha estructurado en cuatro ámbitos de acción: misiones, personas, fortalecimiento de las instituciones, y entorno. Nuestras Universidades se hallan inmersas en la compleja adaptación académica al Espacio Europeo de Educación Superior (EEES). Sin embargo, aunque los campus españoles no están preparados para una metamorfosis de semejante envergadura, esta ineludible cuestión no está siendo prioritaria para Administraciones o Universidades (Campos CalvoSotelo, 2011, 2014; Campos Calvo-Sotelo y Márquez, 2016). En la investigación que se presenta se quiere reflexionar sobre los "espacios sociales de aprendizaje", considerados como espacios docentes no formales, donde los estudiantes adquieren unas competencias y habilidades que son las más demandadas por el mercado laboral. Se trata de proporcionar a los espacios universitarios tradicionales, espacios idóneos para la innovación docente en entornos sociales más informales pero cruciales de cara a la adquisición de las competencias necesarias para el aprendizaje, creando espacios para pensar, formular, presentar, debatir, colaborar, negociar, investigar, etc. Dicha cuestión no va necesariamente ligada a espacios físicos, aunque estos también son importantes para generar las dinámicas que se pretenden con las actividades.

La transformación de los campus ante el EEES obliga a investigar modalidades de aprendizaje alternativas a la clásica lección magistral. Estos modernos formatos pedagógicos necesitan unos espacios "didácticos" ad hoc que los alberguen (Campos Calvo-Sotelo, 2014). La educación superior se enfrenta pues a un escenario esperanzador, pero que exige revolucionar sus modelos (Ripollés, 2001). Todo ello acaba afectando al campus universitario, tanto a la manera cómo estas actividades tienen una repercusión en las clases tradicionales, como en los espacios físicos y virtuales de la universidad (Campos Calvo-Sotelo, 2011). Desde este punto de vista, los espacios sociales de aprendizaje afectan tanto a la imagen como al cuerpo de la institución universitaria.

Así, detrás del concepto definido por "espacio social de aprendizaje" coexisten un conjunto de visiones, estudiadas desde diferentes ámbitos ligados al desarrollo de competencias y el desarrollo de las personas (Alles, 2005; Pereda y Berrocal, 2011). El proyecto de los espacios sociales de aprendizaje en la Universidad Española comenzó a desarrollarse desde la Secretaría General de Universidades del Ministerio de Educación, como parte del cambio de modelo educativo propuesto por el proceso de Bolonia que quiere transitar hacia un sistema basado en la actividad de los propios estudiantes. En este sentido, los espacios sociales de aprendizaje en la Universidad Española los conforman todos los espacios que no son los aularios y que ayudan a completar el aprendizaje formal, en la mayoría de casos con actividades emprendedoras. Espacios que son punto de encuentro entre los diversos colectivos de la Universidad y potencian la comunicación, el desarrollo personal y la convivencia entre estos. Espacios que favorecen, a su vez, la transmisión y difusión del conocimiento multidisciplinar y el intercambio de ideas. Por lo tanto, los espacios sociales de aprendizaje ya existen de forma embrionaria en 
todos los campus: bibliotecas, cafeterías, vías de acceso, zonas verdes, comedores, puntos de atención al estudiante, salas de estudio, pasillos y salas de informática. Pero en este sentido, estos espacios ya no se definen por palabras como aula, sala de conferencias, sala de tutoría, sino con palabras que definen el carácter y el ambiente: espacios para compartir, espacios para pensar, espacios para diseñar, espacios para presentar, para debatir, para colaborar, para negociar o para investigar. Así los nuevos métodos de aprendizaje requieren un amplio menú de espacios:

- $\quad$ Espacios de brainstorming. espacios para pensar y generar ideas, sobre todo en grupos;

- Espacios para el diseño de proyectos: espacios en los que ordenar, dar forma, estructura, contexto y orden a las ideas;

- $\quad$ Espacios para colaboración y para el trabajo en equipo;

- $\quad$ Espacios para el debate;

- $\quad$ Espacios para realizar presentaciones y negociaciones;

- $\quad$ Espacios para actividades de documentación e investigación.

En este sentido, el maratón de emprendimiento es planteado como un hackathon day, que proviene de la unión de las palabras hackery marathon. Su origen parte de un encuentro de programadores que, durante uno o varios días, se juntaban físicamente para trabajar de manera conjunta en sesiones maratonianas sobre un proyecto informático con una doble finalidad: realizar aportaciones al proyecto y aprender unos de otros en un entorno único y excepcional en el que poderse concentrar en un proyecto común. Actualmente este formato de trabajo es utilizado por multinacionales e instituciones para solucionar problemas en el ámbito de la empresa o de la realidad social. A través de grupos de trabajo con distintos objetivos que trabajan de manera ininterrumpida, se pueden aportar soluciones a las problemáticas más diversas. Por lo tanto, se trata de un formato innovador, pero cada vez es más frecuente su uso (Baccarne, Van Comperolle y Mechant, 2015). Por ejemplo, los hackathons más recientes y reconocidos han sido realizados por entidades como Facebook, Google, Microsoft, Hewlett Packard, Syntel, entre otras, y en la ciudad de Barcelona, por parte del propio Ayuntamiento de la ciudad, y empresas y organismos públicos y privados, como Nokia durante la Mobile World Congress de 2013, Vueling en 2014, y otros.

En el caso del BCN Thinking Challenge se trata de un hackathon day que tiene lugar en la Universitat Abat Oliba CEU organizado por los estudiantes de la Universidad que actúan a través del club de emprendedores, que a su vez puede entenderse como otro espacio alternativo de aprendizaje. El maratón se organiza constituyendo una media de seis grupos de ocho personas: cinco alumnos de diferentes universidades y diferentes carreras universitarias y tres profesionales de distintos sectores para transmitir su experiencia. A lo largo del día, cada grupo debe resolver un caso de emprendimiento 
con un objetivo social. La temática del proyecto a desarrollar se da a todos los grupos en el mismo momento al inicio de la jornada laboral.

Durante el día de trabajo, los participantes cuentan con el asesoramiento permanente de profesionales en los ámbitos jurídico-legal, fiscal, laboral, de marketing y contable. Por otro lado, un grupo de headhunters presencia el trabajo de todos los equipos en busca de talento universitario, viendo los progresos de los diferentes grupos de trabajo. Al finalizar el día, doce o más horas después, los distintos equipos deben enviar a la organización sus proyectos de emprendimiento social finalizados.

Al día siguiente, contando con la presencia de un jurado de prestigio profesional, se procede a la entrega de premios, en una gala organizada también por los estudiantes, y que se celebra en el aula magna de la Universidad. En dicha jornada, los equipos de trabajo tienen la oportunidad de exponer sus proyectos al auditorio. El jurado, que tiene en cuenta tanto los proyectos como la exposición y comunicación de los mismos, hace entrega de un premio al mejor proyecto y al mejor emprendedor universitario, seguido de un cóctel donde se promueve el networking entre los participantes.

Con la difusión de esta buena práctica, el presente estudio quiere contribuir a mejorar la calidad universitaria, ya que la difusión y el fomento de actividades formativas como esta, que son ejemplo de espacios alternativos de aprendizaje, forman a los estudiantes en las competencias que más valora el mercado laboral (Prensky, 2015). Ello genera dinámicas de innovación que perfeccionan la función docente, optimizan la adaptación al espacio europeo de educación superior e incrementan la competitividad internacional, pero, sobre todo, forman a los estudiantes en competencias de empleabilidad y emprendimiento que el mercado laboral demanda prioritariamente y son más difíciles de adquirir en el aula.

En el marco europeo, los programas educativos se dirigen a desarrollar en los alumnos un perfil de egreso que en muchos sentidos se relaciona con un perfil de empleabilidad; un perfil para conseguir y conservar un empleo, o bien mejorar y promocionar en su trabajo y la capacidad de adaptación al cambio con una combinación de competencias instrumentales, interpersonales y sistémicas que los espacios sociales de aprendizaje facilitan. Paralelamente, las competencias de profesional experto se desarrollan mediante la práctica y la experiencia acumuladas, relacionadas con otro tipo de competencias ligadas a los contenidos de las asignaturas de los estudiantes (Vargas, 2008).

El desarrollo de esta jornada ayuda a los estudiantes que participan en el Barcelona Thinking Challenge a desarrollar habilidades personales fundamentales en cualquier actividad profesional. Se trata de habilidades como la creatividad, la capacidad de resolución, el trabajo en equipo, el liderazgo, el esfuerzo, la operatividad, la organización, etc. Por supuesto, también tienen ocasión de poner en práctica todos los conocimientos que hayan aprendido durante su vida académica sobre finanzas, recursos humanos, gestión estratégica, logística, retórica, derecho, publicidad, marketing, y un largo etcé- 
tera de asignaturas, pues al ser una actividad transversal cuenta con participantes procedentes de todas las carreras del ámbito social (economistas, técnicos en marketing, logística, periodistas, abogados, psicólogos, educadores, etc.) y técnicas (ingenieros industriales, ingenieros en telecomunicaciones, arquitectos, etc.). Además, los estudiantes disfrutan de la oportunidad de exponerse a la mirada de diferentes empresas que participan en el hackathon day, con la posibilidad de demostrar su valía y poder abrir puertas profesionales que de otra manera permanecerían cerradas.

Por lo que se refiere a la sociedad en su conjunto, se pretende aportar valor, fijando la temática de este tipo de proyectos al ámbito social. Por ejemplo, en la primera edición del Barcelona Thinking Challenge el proyecto consistía en ofrecer una solución viable al problema del desempleo de larga duración entre los parados de Barcelona. La siguiente edición tuvo como objetivo la integración al trabajo de personas con discapacidad. El tercer año se dedicó a la problemática del fracaso escolar. En el caso de la cuarta edición, analizada en este estudio, el tema propuesto fue cómo gestionar óptimamente la convivencia generacional en las empresas. Todas las propuestas son públicas una vez finalizada la entrega de premios, y cualquier persona interesada puede acceder a ellas para hacerlas suyas y llevarlas a cabo.

Por lo tanto, el tema que se plantea en este estudio parte de la premisa de la importancia de las competencias emocionales en la formación integral del estudiante universitario en general y del área de empresa y economía en particular. Una de las tesis es la conveniencia de que las Universidades dispongan de un bagaje fundamentado de modelos de vinculación entre los procesos de enseñanza y las competencias que más se aprecian en los procesos de selección contemporáneos (Pallicer, 2009). Existen espacios alternativos de aprendizaje donde el alumnado universitario adquiere las principales competencias que requiere el mercado de trabajo. Algunos autores lo ligan incluso al uso de espacios físicos concretos (Campos Calvo-Sotelo, 2016), aunque en este trabajo se vincula a actividades específicas, en la línea defendida por otras escuelas que ponen el énfasis en las actividades más que en los espacios (Wagner, 2014), aunque no cabe duda de que disponer de espacios físicos para este tipo de actividades es el complemento ideal de la propia actividad. La promoción de este tipo de actividad generadora de competencias de empleabilidad que fomenta la interacción entre estudiantes, entre estudiantes y profesores, entre estudiantes e investigadores, entre técnicos y estudiantes, pretende la mejora de la adquisición de competencias no formales o inducidas: aprender a compartir, a discutir, a movilizar el espíritu crítico, a utilizar nuevas tecnologías de la comunicación, etc. Nuestra posición parte del denominado learning by doing o aprender haciendo, y por ello creemos que es importante dar difusión a buenas prácticas como el Barcelona Thinking Challenge, como ejemplo de actividades y espacios alternativos de aprendizaje que se han demostrado muy exitosos en la adquisición de competencias de los estudiantes de diferentes disciplinas de la Universidad. 


\section{Metodología}

\subsection{Objetivos}

La investigación se plantea desde tres objetivos diferentes: por un lado, se pretende describir la actividad emprendedora del Barcelona Thinking Challenge como buena práctica del aprender haciendo, como modelo concebido y desarrollado por los propios estudiantes. En segundo lugar, analizar cuál ha sido la visión de este maratón de emprendimiento social entre los participantes de la última edición; y, por último, se desea detectar qué competencias han desarrollado los participantes en esta actividad como espacio no formal de aprendizaje.

\subsection{Procedimiento}

En el caso que nos ocupa, el Barcelona Thinking Challenge, los espacios físicos para realizar la actividad emprendedora se han encontrado optimizando el espacio de la Universidad Abat Oliba CEU. El claustro modernista de la Universidad, donde no se imparten clases, se ha utilizado para colocar grandes mesas en grupo para equipos de hasta diez personas. En el patio central de la universidad al aire libre se han habilitado carpas para poder disfrutar de sombra y poder ubicar mesas y sillas para los grupos de trabajo. Se han facilitado ordenadores personales a los diferentes grupos de trabajo y se ha contratado un catering para atender a los participantes en la maratoniana jornada de un día completo de trabajo, más de 12 horas seguidas de brainstorming y desarrollo de proyectos.

En general, durante ese día, todos los espacios de la Universidad se han puesto al servicio de los estudiantes emprendedores que han trabajado en los proyectos del Barcelona Thinking Challenge, junto a los profesionales participantes en las terrazas, claustros, pasillos, rincones y cafetería. Además, toda la gestión y búsqueda de patrocinios para que la actividad se haya podido celebrar ha corrido a cargo de los estudiantes organizadores del club de emprendedores, con toda la adquisición de competencias que conlleva la organización de este tipo de actividades. Los estudiantes han buscado patrocinadores, por ejemplo para el alquiler de carpas, para el catering, para la gala, etc.

Una vez dispuestos los recursos, son los mismos estudiantes quienes han contratado a las empresas necesarias para lograr la realización de la actividad. La organización de los estudiantes, normalmente articulada a través de comisiones, genera indudablemente la adquisición múltiple de competencias en todo el proceso de generación y gestión de la actividad. Para medir cuantitativamente el grado de adquisición de dichas competencias se ha realizado por primera vez una encuesta de la actividad. 


\subsection{Método}

La investigación realizada se puede enmarcar dentro de un estudio más amplio ex post facto de carácter descriptivo-comparativo donde se han analizado los datos procedentes de la encuesta online (instrumento de análisis) que integra la información sobre la visión de los participantes en este maratón de emprendimiento social.

El cuestionario abordaba preguntas relativas al perfil personal, académico y laboral, competencias desarrolladas y valoración general de la actividad. Se propusieron un total de 16 indicadores. Se pretenden conocer las características de los participantes, y cuál es su visión del desarrollo de determinadas competencias en actividades ubicadas en espacios no formales de aprendizaje.

Las preguntas se han elaborado a partir de cuestionarios ya utilizados en otras investigaciones. El cuestionario fue presentado, en primer lugar, a un grupo de expertos compuesto por profesores especialistas en la materia analizada, en el campo estadístico. Posteriormente se validó por consultores de empresas. Resultado de esta revisión, se realizaron las modificaciones recomendadas y se pasó el cuestionario a la población diana.

\subsection{Población}

La población de estudio está constituida por los 50 estudiantes que han participado en esta cuarta edición del Barcelona Thinking Challenge. Se han obtenido un total de 46 respuestas lo que supone una muestra representativa, para un coeficiente de fiabilidad del 95,5\% y un margen de error del $+/$ - 10\%, trabajando con una relación de p y q del $50 \%$, que representa la situación más desfavorable.

\subsection{Análisis de datos}

Para el tratamiento y análisis de los datos se ha utilizado el programa informático IBM SPSS Statistics, en su versión 20.0. Se han realizado los siguientes análisis: descriptivos univariados de todas las variables implicadas, descriptivos bivariados para explorar la relación entre las variables y pruebas de contraste no paramétricas para determinar la significatividad de las diferencias encontradas.

\section{Resultados}

\subsection{Perfil de los participantes}

Para situar nuestro análisis correctamente, parece adecuado definir primero algunas características del perfil de los individuos que han participado en esta cuarta edición del Barcelona Thinking Challenge. 
En esta edición han participado un total de 50 personas de las que han contestado la encuesta un total de 46 participantes, en su mayoría estudiantes universitarios. La evidencia empírica señala el perfil de un 78,3\% de estudiantes hombres, solteros y mayoritariamente con formación universitaria (82,6\%). El 90\% de los estudiantes tienen una edad inferior o igual a 22 años. Respecto a la situación laboral, los datos indican que el 54,3\% estudia, seguido de un 45,7\% que simultanea sus estudios con alguna dedicación laboral, incluyendo aquí los profesionales participantes.

Así, cuando se analiza su situación familiar en cuanto a con quién viven, el 65,2\% manifiesta vivir con sus progenitores frente al 30,4\% que indica vivir con amigos.

También los datos señalan que el 84,8\% de los participantes no había asistido con anterioridad al Barcelona Thinking Challenge ni a ninguna otra actividad que pueda considerarse un "espacio alternativo de aprendizaje". En este sentido, el 47,8\% ha conocido la existencia de la actividad a través de la institución, seguido de un $26,1 \%$ que indica que ha sido un profesor el que ha hecho llegar la información del mismo.

\subsection{Competencias desarrolladas en el BCN Thinking Challenge}

Para analizar en qué medida el Barcelona Thinking Challenge ha permitido desarrollar determinadas competencias, se han considerado las siguientes recogidas en la Tabla 1.

Tabla 1. Relación de competencias trabajadas durante el Barcelona Thinking Challenge

\begin{tabular}{|l|}
\hline Competencias \\
\hline Ser capaz de aplicar sus conocimientos a la resolución de problemas \\
\hline Ser capaz comunicar información, ideas, problemas y soluciones de forma oral \\
\hline Ser capaz comunicar información, ideas, problemas y soluciones de forma escrita \\
\hline Ser capaz de emprender y culminar proyectos \\
\hline Ser capaz de participar en equipos de trabajo y liderarlos \\
\hline Ser capaz de negociar y gestionar acuerdos mediante la elaboración de argumentos \\
\hline Ser capaz de innovar en el sector de la empresa \\
\hline
\end{tabular}

Fuente: Elaboración propia

Los resultados indican que según la opinión de los participantes en este maratón de emprendimiento social, el Barcelona Thinking Challenge ha permitido desarrollar un conjunto de competencias muy valoradas por el mercado laboral. La competencia más valorada por los participantes con un $72 \%$ es la competencia de ser capaz de comunicar información, ideas, problemas y soluciones de forma oral. Tras un día intensivo de trabajo en el que se elabora el proyecto, este tiene que comunicarse a un amplio auditorio y un jurado especializado, por lo que la preparación de ligar el proyecto realizado con su 
presentación es una tarea altamente valorada por los participantes. Ello va unido con el reconocimiento de la importancia que los participantes le dan a ser capaz de innovar en el sector de la empresa, con un 30\%, y ser capaz de comunicar información, ideas, problemas y soluciones de forma escrita, con un $28 \%$.

Le sigue en valoración, con un 65\%, la competencia de ser capaz de participar en equipos de trabajo y liderarlos. Los participantes son conscientes de la importancia en el mundo laboral del trabajo en equipo. Por su parte la competencia de ser capaz de negociar y gestionar acuerdos mediante la elaboración de argumentos, ligada al trabajo en equipo, es valorada por el $48 \%$ de los participantes. Ser capaz de aplicar los conocimientos a la resolución de problemas es valorado por un 59\% de los participantes. Ello se liga a la visión tradicional del desarrollo de competencias en el aula y va seguido por una valoración del $52 \%$ de la competencia ser capaz de emprender y culminar proyectos.

En referencia a la valoración general del Barcelona Thinking Challenge como espacio alternativo de aprendizaje, los individuos participantes valoran este maratón con una media de 4,16 de una escala de 1 a 5. Por lo tanto, los datos señalan una gran satisfacción en la participación de este tipo de actividades no formales que configuran un espacio alternativo de aprendizaje.

Respecto a qué mejoras se podrían introducir en próximas ediciones del Barcelona Thinking Challenge, los datos señalan que mayoritariamente se desearía que la ratio de grupos de trabajo fuera más pequeña y tener más tiempo y más herramientas de mentoring durante el proceso del hackathon day. A modo resumen, se recoge en la Figura 1 el impacto o valoración de las competencias más desarrolladas en este hackathon day desde la visión de los participantes.

Figura 1. Distribución según valoración de las competencias desarrolladas durante el Barcelona thinking challenge

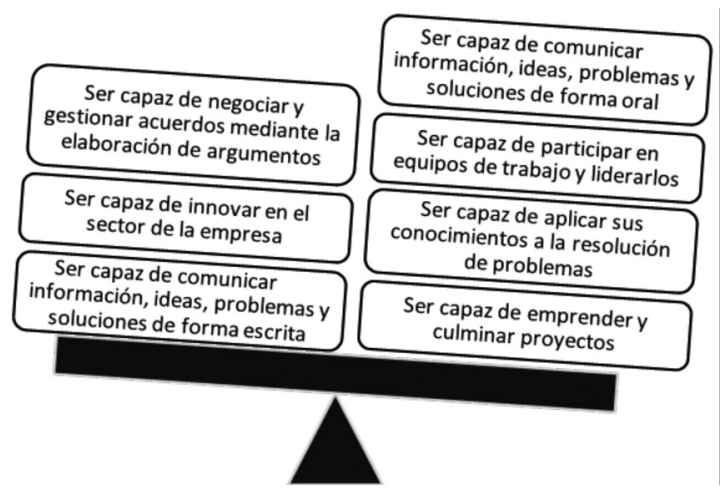

Fuente: Elaboración propia 


\section{Conclusiones}

En una actividad como el Barcelona Thinking Challenge se observa la necesidad de redefinir los espacios sociales de aprendizaje como ampliación de la diversidad de espacios y escenarios para fomentar las nuevas y distintas pedagogías y la adquisición de competencias de forma complementaria a la formación tradicional que se basan en actividades emprendedoras (Rivas, 2014), pensando en actividades del aprendizaje y sus escenarios (y no en meros lugares físicos); pensando en el aprendizaje formal y también en el aprendizaje informal; analizando dónde y cómo sucede el proceso de adquisición de conocimientos, y centrando el enfoque en los estudiantes.

En este sentido, en esta investigación se ha analizado esta actividad específica donde los estudiantes desarrollan sus competencias. Así, los espacios donde el aprendizaje tiene lugar ejercen un impacto directo sobre el aprendizaje en sí (Barrett, Zhang, Moffat y Kobbacy, 2013), de modo que el éxito y progresión de la enseñanza dependen en gran modo de esos espacios. Por ello, los espacios informales de aprendizaje en la educación superior contribuyen de manera significativa a motivar a los estudiantes a desarrollar sus competencias (Harrop y Turpin, 2013), especialmente sus competencias emprendedoras (Pallicer, 2009).

En este sentido, esta investigación ha analizado una actividad específica, el maratón de emprendimiento Barcelona Thinking Challenge, donde los estudiantes han podido desarrollar determinadas competencias. Dicha actividad ha entrado en su cuarta edición con un gran éxito de seguimiento y de consecución de los objetivos propuestos. El hecho de colaborar en seis grupos formados por ocho personas (cinco alumnos de diferentes universidades y tres profesionales de distintos sectores) hace posible el trabajo en equipo en un proyecto común. A lo largo del día, cada equipo debe resolver un caso de emprendimiento con un objetivo social. Los resultados indican que, según la opinión de los participantes en este maratón de emprendimiento social, se han podido desarrollar un conjunto de competencias muy valoradas por el mercado laboral (Barraycoa y Lasaga, 2011).

Estos resultados parten de un proyecto de investigación más amplio y permiten destacar que los participantes valoran con alta intensidad este maratón de emprendimiento social. Los resultados muestran un alto desarrollo en las competencias relacionadas con ser capaz de participar en equipos de trabajo, ser capaz de liderarlos y poder aplicar los conocimientos adquiridos en la universidad a la resolución de problemas. Así, comunicar ideas, problemas y soluciones de forma oral es una de las competencias que los participantes valoran como mejor adquiridas durante la maratón de emprendimiento.

Los datos señalan una gran satisfacción en la participación de este tipo de actividades no formales que configuran un espacio alternativo de aprendizaje. Como señala Prensky (2015) los participantes son conscientes de la importancia en el mundo laboral del trabajo en equipo, y la evidencia lo ha corroborado. Actualmente la universidad ha dejado de ser el 
único lugar donde ocurre el aprendizaje y tampoco puede pretender asumir por sí sola la función educacional de la sociedad; es aquí donde cobra importancia el aprendizaje que se desarrolla en contextos no formales, generando así oportunidades para el aprendizaje permanente y de calidad para toda la comunidad, haciendo de la educación una necesidad y una tarea de todos.

En suma, se puede decir que los contextos de aprendizaje se van construyendo a lo largo de la vida de las personas; la escuela, la familia, las actividades extracurriculares son fuentes de variados aprendizajes. De este modo, los distintos tipos de contextos deben posibilitar la comunicación y el encuentro con las personas, dando a lugar a materiales y actividades que estimulen la curiosidad, la capacidad creadora y el diálogo; permitiendo la expresión libre de las ideas, intereses, necesidades y estados de ánimo de todos y en una relación ecológica con la cultura y la sociedad en general (Duarte, 2003).

\section{Bibliografía}

Alles, M. (2005): Dirección estratégica de Recursos Humanos: gestión por competencias. México: Ediciones Granica.

Baccarne, B., Van Comperolle, M., \& Mechant, P. (2015): Exploring hackathons: civic vs. Product innovation hackathons. i3 conference 2015: participating in innovation. Innovating in participation Paris. Disponible en: http://hdl.handle.net/1854/ LU-7033553.

Barraycoa, J., y Lasaga, O. (2011): El camino hacia el empleo en un mundo complejo. Zaragoza: Fragua.

Barrett, P., Zhang, Y., Moffat, J., \& Kobbacy, K. (2013): “A holistic, multi-level analysis identifying the impact of classroom design on pupils' learning”, Building and Environment, n. 59, pp. 678-689.

Campos Calvo-Sotelo, P. (2011): La evolución histórica del espacio físico de la Universidad: Impulsos conceptuales, paradigmas arquitectónicos, estrategias institucionales y propuestas recientes de innovación. Universidad Carlos III de Madrid.

Campos Calvo-Sotelo, P. C., y Márquez, F. C. (2016): "Memoria e innovación en los espacios físicos de enseñanza/aprendizaje de la educación superior. La transformación del límite como respuesta de la Arquitectura a la innovación docente”, Historia y Memoria de la Educación, n. 3, pp. 279-320.

Calvo-Sotelo, P. C. (2014): "Innovative educational spaces: architecture, art and nature for university excellence", Aula: Revista de Pedagogía de la Universidad de Salamanca, n. 20, pp. 159-174.

Duarte, J. (2003): “Ambientes de aprendizaje una aproximación conceptual”, Revista Iberoamericana de Educación, pp. 118. Disponible en: http://www.rieoei.org/deloslectores/524Duarte 
Harrop, B., \& Turpin, B. (2013): “A Study Exploring Learners' Informal Learning Space Behaviors, Attitudes, and Preferences”, New Review of Academic Librarianship, n. 19 (1). DOI: 10.1080/13614533.2013.740961

Pallicer, C. (2009): La evaluación de las competencias básicas. Madrid: Ediciones PPC.

Prensky, M. (2015): El mundo necesita un nuevo currículo: habilidades para pensar, crear, relacionarse y actuar. Madrid: Ediciones SM.

Pereda, S., y Berrocal, F. (2011): Dirección y Gestión de Recursos Humanos por Competencias. Madrid: Centro de Estudios Ramón Areces.

Ripollés, M. (2001): “Aprender a emprender en las universidades”, ARBOR Ciencia, Pensamiento y Cultura, n. 187, pp. 124156.

Rivas, M. (2014): Innovación educativa. Teoría, procesos y estrategias. Madrid: Síntesis.

Vargas, M. (2008): Perfiles de empleabilidad y desempeño profesional, Ponencia presentada al IX Congreso Nacional de Investigación Educativa. Disponible en: http://www.comie.org.mx/congreso/memoriaelectronica/v09/ponencias/at10/ PRE1178332449.pdf

Wagner, T. (2014): Creando innovadores: la formación de los jóvenes que cambiarán el mundo. Madrid: Kolima. 